\title{
Transformation Optics Approach for Goos-Hänchen Shift Enhancement at Metamaterial Interfaces
}

\author{
Lieve Lambrechts ${ }^{a}$, Vincent Ginis $^{a}$, Jan Danckaert $^{a}$ and Philippe Tassin ${ }^{b, a}$ \\ ${ }^{a}$ Applied Physics Research Group, Vrije Universiteit Brussel, \\ Pleinlaan 2, B-1050 Brussel, Belgium \\ ${ }^{b}$ Department of Physics, Chalmers University, SE-412 96, Göteborg, Sweden
}

\begin{abstract}
Since its first observation in 1947, the Goos-Hänchen effect-an electromagnetic wave phenomenon where a totally reflected beam with finite cross section undergoes a lateral displacement from its position predicted by geometric optics - has been extensively investigated for various types of optical media such as dielectrics, metals and photonic crystals. Given their huge potential for guiding and sensing applications, the search for giant and tunable Goos-Hänchen shifts is still an open question in the field of optics and photonics. Metamaterials allow for unprecedented control over electromagnetic properties and thus provide an interesting platform in this quest for Goos-Hänchen shift enhancement. Over the last few years, the Goos-Hänchen effect has been investigated for specific metamaterial interfaces including graphene-on-dielectric surfaces, negative index materials and epsilonnear-zero materials. In this contribution, we generalize the approach for the investigation of the Goos-Hänchen effect based on the geometric formalism of transformation optics. Although this metamaterial design methodology is generally applied to manipulate the propagation of light through continuous media, we show how it can also be used to describe the reflections arising at the interface between a vacuum region and a transformed region with a metamaterial implementation. Furthermore, we establish an analytical model that relates the magnitude of the Goos-Hänchen shift to the underlying geometry of the transformed medium. This model shows how the dependence of the Goos-Hänchen shift on geometric parameters can be used to dramatically enhance the size of the shift by an appropriate choice of permittivity and permeability tensors. Numerical simulations of a beam with spatial Gaussian profile incident upon metamaterial interfaces verify the model and firmly establish a novel route towards Goos-Hänchen shift engineering using transformation optics.
\end{abstract}

Keywords: Metamaterials, metamaterial interfaces, metasurfaces, transformations optics, Gaussian beam displacement, Goos-Hänchen effect, Goos-Hänchen shift

\section{INTRODUCTION}

In 1968, Victor Veselago first introduced the notion of negative index materials, exhibiting simultaneous negative permittivity and permeability resulting in a negative refractive index as a theoretical artefact. ${ }^{1}$ Contrary to materials readily found in nature, such materials would imply refraction to the opposite side of the normal, known as negative refraction. The theoretical work of John Pendry published in 1996 and 1999 demonstrated how synthetic materials could be designed exhibiting both negative permittivity and negative permeability using metallic microstructures, thus making the theoretical prediction of Veselago a possible reality. ${ }^{2,3}$

Not long after, in 2000, the first left-handed 'metamaterial' was experimentally demonstrated by David Smith: a composite medium consisting of a periodic array of interspaced conducting nonmagnetic split-ring resonators and continuous wires generating negative effective permeability and negative effective permittivity, respectively. ${ }^{4}$ Such an artificial left-handed medium would exhibit unique electromagnetic properties not found in nature. ${ }^{5,6}$ Veselago already realised that refraction to the opposite side of the normal implies the possiblity of constructing a flat lens. This was further explored by Pendry later that year, who realised that Veselago's lens could not only focus light, but would allow imaging beyond the diffraction limit. ${ }^{7}$ This spiked the interest of many research groups, intent on experimental verification of negative refracting media in quest for the perfect lens. A first

Further author information: (Send correspondence to Lieve Lambrechts)

Lieve Lambrechts: E-mail: lieve.lambrechts@vub.ac.be, Telephone: +32 26293653

Metamaterials X, edited by Allan D. Boardman, Nigel P. Johnson, Kevin F. MacDonald, Ekmel Özbay, Proc. of SPIE Vol. 9883, 98831B · @ 2016 SPIE · CCC code: 0277-786X/16/\$18 · doi: 10.1117/12.2227519 
successful experiment displaying negative refraction was set up by Richard Shelby and his colleagues in $2001 .^{8}$ The experiment was later repeated and its results confirmed by Andrew Houck and his team at MIT. ${ }^{9}$ Figure 1 shows the constructed metamaterial, implementing structured arrays of split-ring resonators, which give rise to a negative effective permeability and straight wires, which provide negative effective permittivity.

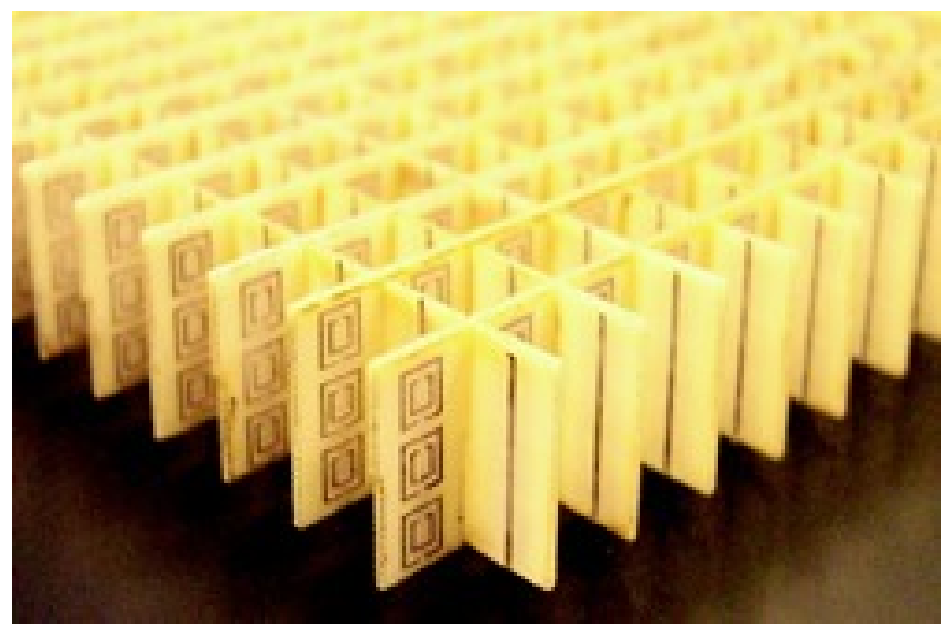

Figure 1: Photograph of the metamaterial structure used in 2003 by Houck et al. to demonstrate negative refraction. Negative effective permeability is provided by the periodic array of square split-ring resonators, whereas the arrays of straight wires provide negative effective permittivity. Reprinted figure with permission from Ref. 7. Copyright (2003) by the American Physical Society.

In the following decade, further research showed - what is probably just a first glimpse of - the wide range of designer electromagnetic response made possible by the use of artificially constructed metamaterials. ${ }^{10-12}$ By building a material consisting of structured arrays of subwavelength resonant structures, which emulate the role of atoms in matter, the material will behave as a homogenous medium described by an effective refractive index. By engineering the properties of the individual building blocks as well as their periodic arrangement throughout the material, one can achieve a desired electromagnetic response.

\section{TRANSFORMATION OPTICS}

To design metamaterials exhibiting a specific electromagnetic response, one needs a design procedure that can translate the desired effect on electromagnetic waves into the material parameters needed for the metamaterial medium. Transformation optics is a convenient tool in this regard. Since its theoretical conception in 2006 transformation optics has already shown great versatility in controlling optical properties. At first, transformation optics has allowed for the design of metamaterial devices that modify the trajectory of light, such as beam benders, beam splitters and invisibility cloaks. ${ }^{13-18}$ Furthermore, several applications have been devised which manipulate electromagnetic fields up to an unprecedented level, going far beyond the modification of light ray trajectories. ${ }^{19-30}$

In 2006 Ulf Leonhardt and John Pendry independently came to the realisation that the form invariance of Maxwell's equations under coordinate transformations can be used to identify an equivalence between a medium with traditional electromagnetic material parameters, expressed on the background of a curved coordinate system, and a material with exotic material parameters, expressed in a traditional (Cartesian) coordinate system. ${ }^{31,32}$

Following Einstein's general relativity, we know that the presence of large masses introduces curvature in the fabric of spacetime. In this curved spacetime, light rays will propagate along the curved coordinate lines or geodesics. This bending of light bears an analogy to the bending of light that occurs when light propagates through a medium with an inhomogeneous refractive index profile. In this case, light will choose a path of extremal optical path length according to Fermat's principle, resulting in a curved trajectory through the inhomogeneous medium. 
This analogy becomes more apparent when considering Maxwell's equations describing the propagation of light in both cases. First,

$$
\begin{gathered}
\pm[i, j, k] \frac{\partial E_{k}}{\partial x^{j}}=-\frac{\partial \sqrt{g} g^{i j} \mu_{0} H_{j}}{\partial t}, \\
\pm[i, j, k] \frac{\partial \mu_{0} H_{k}}{\partial x^{j}}=\varepsilon_{0} \mu_{0} \frac{\partial\left(\sqrt{g} g^{i j} E_{j}\right)}{\partial t}
\end{gathered}
$$

show Maxwell's equations in empty space, expressed on the background of a curved coordinate system specified by the metric tensor $g_{i j}$. Here, $g=\operatorname{det}\left(g_{i j}\right)$ and $[i, j, k]$ is the permutation symbol, defined by $[i, j, k]=1$ if $(i j k)$ is an even permutation of (123), $[i j k]=1$ if $(i j k)$ is an odd permutation of $(123)$, and $[i j k]=0$ for all other combinations of the triplet $(i j k)$. Second,

$$
\begin{gathered}
{[i, j, k] \frac{\partial E_{k}}{\partial x^{j}}=-\frac{\partial \mu_{0} \mu^{i j} H_{j}}{\partial t},} \\
{[i, j, k] \frac{\partial \mu_{0} H_{k}}{\partial x^{j}}=\varepsilon_{0} \mu_{0} \frac{\partial\left(\varepsilon^{i j} E_{j}\right)}{\partial t}}
\end{gathered}
$$

describe Maxwell's equations in a specific material with electric permittivity and magnetic permeability tensors $\varepsilon^{i j}$ and $\mu^{i j}$, expressed in a regular Cartesian coordinate system.

An equivalence relation can be established between these sets of equations, relating the metric tensor $g_{i j}$ describing the curved coordinate system to electric permittivity and magnetic permeability tensors $\varepsilon^{i j}$ and $\mu^{i j}$ as follows:

$$
\varepsilon^{i j}=\mu^{i j}= \pm \sqrt{g} g^{i j}
$$

This equivalence relation can then be used to design macroscopic metamaterials that allow for enhanced manipulation of electromagnetic waves based on geometrical deformations. ${ }^{33,34}$

The design procedure usually consists of three steps, as visualized in Fig. 2. We start from light propagation in an empty space expressed in a Cartesian coordinate system [see Fig. 2(a)]. Subsequently, we transform the path of the light ray in a desired way, by changing the coordinate system as shown in Fig. 2(b). Using the equivalence relations of transformation optics, we then obtain the electromagnetic parameters of the specific (meta)material in which light propagates along this desired path [see Fig. 2(c)].

(a)

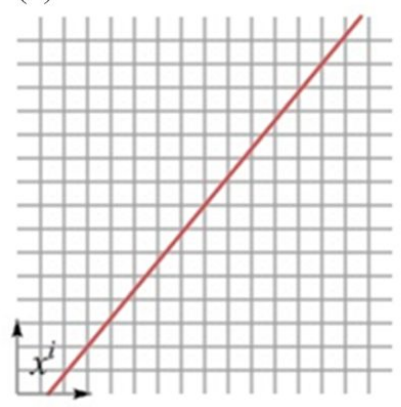

b)

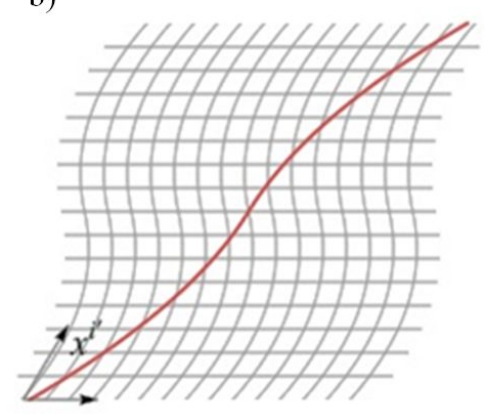

(c)

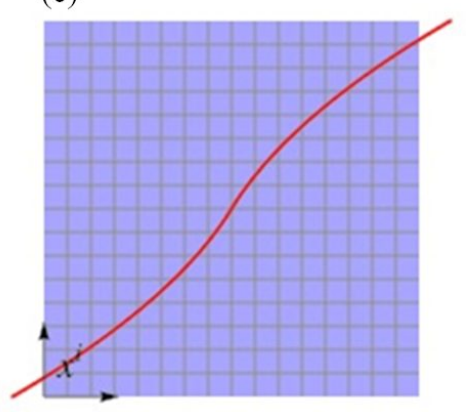

Figure 2: The design procedure of transformation optics: (a) the path of a light ray in empty space expressed in Cartesian coordinates, (b) is transformed under a coordinate transformation. (c) Using the equivalence relations, the effect of this coordinate transformation is implemented in a specific material.

This formalism can thus elegantly be used to transform the path of a light ray in a desired way and subsequently obtain a material implementation from the metric describing the performed coordinate transformation. Recently, there has been a growing interest in using metamaterial interfaces for waveguiding purposes. ${ }^{35} \mathrm{Al}-$ though transformation optics was initially developed for the smooth manipulation of light beam trajectories, we will show in this contribution that it can also be used to understand and design optical phenomena at (meta)material interfaces. 


\section{THE GOOS-HÄNCHEN EFFECT}

The Goos-Hänchen effect is such an interface phenomenon. When an electromagnetic beam with finite cross section totally reflects at the interface between two media, the reflected beam experiences a small shift $s$ along the interface - known as the Goos-Hänchen shift - with respect to the beam predicted by geometric optics (see Fig. 3). This effect was observed experimentally for the first time by Hermann Goos and Hilda Hänchen in 1947, employing multiple total internal reflections in a glass slab, hereby significantly increasing the extremely small shift associated with a single reflection. ${ }^{36}$

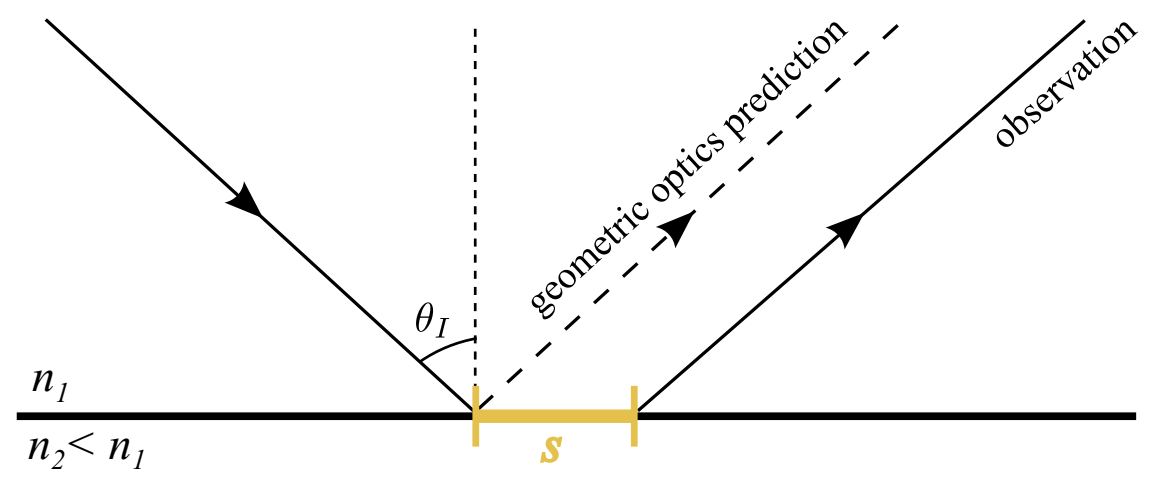

Figure 3: Electromagnetic beams with finite cross section display a lateral shift $s$, known as the Goos-Hänchen shift, when passing from an optically dense medium to an optically less dense medium.

A theoretical explanation of this reflection phenomenon was originally proposed by Kurt Artmann in 1948 using a stationary phase method. ${ }^{37,38}$ Considering the incident beam as the superposition of plane waves that encounter different phase shifts upon reflection, it can be shown that the superposition of these slightly phaseshifted plane waves result in a reflected beam that is laterally displaced along the interface. If $\phi_{r}$ denotes the phase of the complex reflection coefficient $r$, the Goos-Hänchen shift $s$ is given by

$$
s=-\frac{\partial \phi_{r}}{\partial k_{I}^{\|}}=-\frac{1}{k_{I} \cos \theta_{I}} \frac{d \phi_{r}}{d \theta_{I}}
$$

where $k_{I}^{\|}$represents the component of the incident wave vector parallel to the material interface and $\theta_{I}$ is the angle of incidence.

Over the past few decades, the Goos-Hänchen effect has been extensively investigated among all kinds of optical media, such as dielectrics, ${ }^{39-41}$ metals, ${ }^{42,43}$ photonic crystals $^{44,45}$ and metamaterials such as left-handed media ${ }^{46}$ and epsilon-near-zero metamaterials. ${ }^{47}$ Cleverly employing various aspects of this effect has led to several applications in sensing ${ }^{48}$ and waveguiding. ${ }^{49}$ However, an open question remaining in this field is the finding of a scheme to enhance the magnitude of the Goos-Hänchen effect.

In this contribution, we explore a new route towards Goos-Hänchen shift tuning using the geometric paradigm of transformation optics. We illustrate how the Goos-Hänchen shift $s$ can be related to the geometric properties of the reflecting material. This relationship can then be used to dramatically enhance Goos-Hänchen shifts, opening a route towards efficient beam manipulation.

\section{GOOS-HÄNCHEN SHIFT ENHANCEMENT AT METAMATERIAL INTERFACES USING TRANSFORMATION OPTICS}

Transformation optics has been extensively used to manipulate the propagation of electromagnetic beams through continuous media. In our setup, however, we use this technique to describe the physics that arises at the interface between a vacuum region that remains untransformed, and a transformed vacuum region. Applying a firstprinciples approach using Maxwell's equations in combination with the appropriate boundary conditions, we obtained analytical expressions for the (complex) reflection coefficients describing the scattering at the interface 
between an (untransformed) vacuum region and a metamaterial region implementing an isotropic stretching of the coordinates.

Using this analytical model, different reflective regimes can be distinguished, depending on the stretching parameter $a$ of the coordinate transformation and on the angle of incidence $\theta_{I}$. The derived expressions for the reflection and transmission coefficients were verified numerically by full-wave simulations using a finite-elements solver, both for TE and TM polarization.

The steep variation of the phase of the reflection coefficient with respect to the angle of incidence apparent in the regime of total reflection, i.e. for stretching parameters $a<1$, implies the presence of a nontrivial GoosHänchen effect. From the analytical model, one can obtain expressions for the Goos-Hänchen shift $s$, depending on the angle of incidence $\theta_{I}$ for a stretching of the coordinates by $a$. For certain stretching parameters, the minimal Goos-Hänchen shift that can be obtained already exceeds one wavelength, opening up a route towards dramatic Goos-Hänchen shift enhancement.

\section{CONCLUSIONS}

We illustrated how the Goos-Hänchen shift can be related to the geometric properties of the reflecting metamaterial by using the geometrical formalism of transformation optics. The established relationship between the Goos-Hänchen shift $s$ and the parameter of the coordinate stretching $a$ can then be employed to design metamaterials exhibiting dramatically enhanced Goos-Hänchen shifts, thus opening a route towards efficient beam manipulation.

\section{ACKNOWLEDGMENTS}

V. G. acknowledges the Research Foundation Flanders (FWO) for a postdoctoral fellowship. Work at Vrije Universiteit Brussel was also supported by the Research Council (OZR) of the Vrije Universiteit Brussel and

the Interuniversity Attraction Poles programme of the Belgian Science Policy Office, under grant IAP P7-35 "photonics@be".

\section{REFERENCES}

[1] Veselago, V. G., "The electrodynamics of substances with simulaneously negative values of $\varepsilon$ and $\mu$," Sov. Phys. Usp. 10(4), 517-526 (1964).

[2] Pendry, J. B., Holden, A. J., Stewart, W. J., and Youngs, I., "Extremely low frequency plasmons in metallic mesostructures," Phys. Rev. Lett. 76(25), 4773-4776 (1996).

[3] Pendry, J. B., Holden, A. J., Robbins, D. J., and Stewart, W. J., "Magnetism from conductors and enhanced nonlinear phenomena," IEEE Trans. Microw. Theory Techn. 47(11), 2075-2084 (1999).

[4] Smith, D. R., Padilla, W. J., Vier, D. C., Nemat-Nasser, S. C., and Schultz, S. "Composite medium with simultaneously negative permeability and permittivity," Phys. Rev. Lett. 84(18), 4184-4187 (2000).

[5] Foteinopoulou, S., Economou, E. N., and Soukoulis, C. M., "Refraction in media with a negative refractive index," Phys. Rev. Lett. 90(10), 107402 (2003).

[6] McCall, M. W., Lakhtakia, A., and Weiglhofer, W. S., "The negative index of refraction demystified," Eur. J. Phys. 23(3), 353 (2002).

[7] Pendry, J. B., "Negative refraction makes a perfect lens," Phys. Rev. Lett. 85(18), 3966-3969 (2000).

[8] Shelby, R. A., Smith, D. R., and Schultz, S., "Experimental verification of a negative index of refraction," Science 292(5514), 77-79 (2001).

[9] Houck, A. A., Brock, J. B., and Chuang, I. L., "Experimental observations of a left-handed material that obeys Snell's law," Phys. Rev. Lett. 90(13), 137401 (2003).

[10] Silveirinha, M., and Engheta, N., "Tunneling of electromagnetic energy through subwavelength channels and bends using $\varepsilon$-near-zero materials," Phys. Rev. Lett. 97(15), 157403 (2006).

[11] Soukoulis, C. M., and Wegener, M., "Past achievements and future challenges in the development of threedimensional photonic metamaterials," Nature Photon. 5(9), 523-530 (2011). 
[12] Catrysse, P. B., and Fan, S., "Routing of deep-subwavelength optical beams and images without reflection and diffraction using infinitely anisotropic metamaterials," Adv. Mater. 25(2), 194-198 (2013).

[13] Rahm, M., Roberts, D. A., Pendry, J. B., and Smith, D. R., "Transformation-optical design of adaptive beam bends and beam expanders," Opt. Express 16(15), 11555-11567 (2008).

[14] Schurig, D., Mock, J. J., Justice, B. J., Cummer, S. A., Pendry, J. B., Starr, A. F., and Smith, D. R., "Metamaterial electromagnetic cloak at microwave frequencies," Science 314(5801), 977-980 (2006).

[15] Li, J. and Pendry, J. B., "Hiding under the carpet: a new strategy for cloaking," Phys. Rev. Lett. 101, 203901 (2008).

[16] Liu, R., Ji, C., Mock, J. J., Chin, J. Y., Cui, T. J., and Smith, D. R., "Broadband ground-plane cloak," Science 323(5912), 366-369 (2009).

[17] Kanté, B., Germain, D., and de Lustrac, A., "Experimental demonstration of a nonmagnetic metamaterial cloak at microwave frequencies," Phys. Rev. B, 80(20), 201104 (2009).

[18] McCall, M. W., Favaro, A., Kinsler, P., and Boardman, A., "A spacetime cloak, or a history editor," J. Opt. 13, 024003 (2011).

[19] Schurig, D., Pendry, J. B., and Smith, D. R., "Calculation of material properties and ray tracing in transformation media," Opt. Express 14, 97949804 (2006).

[20] Chen, H., and Chan, C. T., "Electromagnetic wave manipulation by layered systems using the transformation media concept," Phys. Rev. B 78(5), 054204 (2008).

[21] Ginis, V., Tassin, P., Craps, B., and Veretennicoff, I., "Frequency converter implementing an optical analogue of the cosmological redshift," Opt. Express 18(5), 5350-5355 (2010).

[22] Zentgraf, T., Liu, Y., Mikkelsen, M. H., Valentine, J., and Zhang, X., "Plasmonic luneburg and eaton lenses," Nature Nanotech. 6(3), 151-155 (2011).

[23] Tichit, P.-H., Burokur, S. N., Germain, D., and de Lustrac, A., "Design and experimental demonstration of a high-directive emission with transformation optics," Phys. Rev. B, 83(15), 155108 (2011).

[24] Ginis, V., Tassin, P., Danckaert, J., Soukoulis, C. M., and Veretennicoff, I. "Creating electromagnetic cavities using transformation optics," New J. Phys. 14(3), 033007 (2012).

[25] Sheng, C., Liu, H., Wang, Y., Zhu, S. N., and Genov, D. A., "Trapping light by mimicking gravitational lensing," Nature Photon. 7(11), 902-906 (2013).

[26] Liu, F., Liang, Z., Li, J., and Pendry, J. B., "Manipulating polarization and impedance signature: A reciprocal field transformation approach," Phys. Rev. Lett. 111, 033901 (2013).

[27] Castaldi, G., Savoia, S., Galdi, V., Alù, A., and Engheta, N., "P T metamaterials via complex-coordinate transformation optics," Phys. Rev. Lett. 110, 173901 (2013).

[28] Ginis, V., Tassin, P., Soukoulis, C. M., and Veretennicoff, I., "Enhancing optical gradient forces with metamaterials," Phys. Rev. Lett. 110(5), 057401 (2013).

[29] Ginis, V., Danckaert, J., Veretennicoff, I., and Tassin, P., "Controlling Cherenkov radiation with transformation-optical metamaterials," Phys. Rev. Lett. 113(16), 167402 (2014).

[30] Ginis, V., and Tassin, P., "Transformation optics beyond the manipulation of light trajectories," Phil. Trans. R. Soc. A 373(2049), 20140361 (2015).

[31] Leonhardt, U., "Optical conformal mapping," Science 312(5781), 1777-1780 (2006).

[32] Pendry, J. B., Schurig, D., and Smith, D. R., "Controlling electromagnetic fields," Science 312(5781), 1780$1782(2006)$.

[33] Leonhardt, U., and Philbin, T. G., "Transformation optics and the geometry of light," Prog. Opt. 53(08), 69-152 (2009).

[34] Chen, H., Chan, C. T., and Sheng, P., "Transformation optics and metamaterials," Nature Mater. 9(5), 387-396 (2010).

[35] Viaene, S., Ginis, V., Danckaert, J., and Tassin, P., "Transforming two-dimensional guided light using nonmagnetic metamaterial waveguides" Phys. Rev. B 93(8), 085429 (2016).

[36] Goos, F., and Hänchen, H., "Ein neuer und fundamentaler Versuch zur Totalreflexion," Ann. Phys. (Berlin) 436(78), 333-346 (1947).

[37] Artmann, K., "Berechnung der Seitenversetzung des totalreflektierten Strahles," Ann. Phys. (Berlin) 437(12), 87-102 (1948). 
[38] Bliokh, K. Y., and Aiello, A., "Goos-Hänchen and Imbert-Fedorov beam shifts: an overview," J. Opt. 15(1), 014001 (2013).

[39] Wild, W. J., and Giles, C. L., "Goos-Hänchen shifts from absorbing media," Phys. Rev. A, 25(4), 2099-2101 (1982).

[40] Lai, H. M., and Chan, S. W., "Large and negative Goos-Hänchen shift near the Brewster dip on reflection from weakly absorbing media," Opt. Lett. 27(9), 680-682 (2002).

[41] Wang, L. G., Chen, H., and Zhu, S. Y., "Large negative Goos-Hänchen shift from a weakly absorbing dielectric slab," Opt. Lett. 30(21), 2936-2938 (2005).

[42] Merano, M., Aiello, A., Van Exter, M. P., Eliel, E. R., and Woerdman, J. P., "Observation of Goos-Hänchen shifts in metallic reflection," Opt. Express 15(24), 15928-15934 (2007).

[43] Leung, P. T., Chen, C. W., and Chiang, H. P., "Large negative Goos-Hänchen shift at metal surfaces," Opt. Commun. 276(2), 206-208 (2007).

[44] Felbacq, D., Moreau, A., and Smaâli, R., "Goos-Hänchen effect in the gaps of photonic crystals," Opt. Lett. 28(18), 1633-1635 (2003).

[45] He, J., Yi, J., and He, S., "Giant negative Goos-Hänchen shifts for a photonic crystal with a negative effective index," Opt. Express 14(7), 3024-3029 (2006).

[46] Shadrivov, I. V., Zharov, A. A., and Kivshar, Y. S., "Giant Goos-Hänchen effect at the reflection from left-handed metamaterials," Appl. Phys. Lett. 83(13), 2713-2715 (2003).

[47] Xu, Y., Chan, C. T., and Chen, H., "Goos-Hänchen effect in epsilon-near-zero metamaterials," Sci. Rep. 5 (2015).

[48] Taya, S. A., El-Farram, E. J., and El-Agez, T. M., "Goos-Hänchen shift as a probe in evanescent slab waveguide sensors," AEÜ Int. J. Electron. Commun. 66(3), 204-210 (2012).

[49] Tassin, P., Sahyoun, X., and Veretennicoff, I., "Miniaturization of photonic waveguides by the use of lefthanded materials," Appl. Phys. Lett. 92(20), 203111 (2008). 